\title{
Comparison of MicroRNA Profiles in Extracellular Vesicles from Small and Large Goat Follicular Fluid
}

\author{
Qiang Ding ${ }^{1,2}{ }^{\circ}$, Miaohan Jin ${ }^{2}(D)$, Peter Kalds ${ }^{2,3}$, Chunhua Meng ${ }^{1}$, Huili Wang ${ }^{1}$, Jifeng Zhong ${ }^{1}$, \\ Xiaolong Wang ${ }^{2, *}$ and Yulin Chen ${ }^{2, *}$ \\ 1 Institute of Animal Science, Jiangsu Academy of Agricultural Sciences, Nanjing 210014, China; \\ dingqiang198907@163.com (Q.D.); mengchunhua@jaas.com.cn (C.M.); wanghuili318@163.com (H.W.); \\ zhongifeng64@sina.cn (J.Z.) \\ 2 Key Laboratory of Animal Genetics, Breeding and Reproduction of Shaanxi Province, College of Animal \\ Science and Technology, Northwest A\&F University, Xianyang 712100, China; \\ jinmiaohan@nwafu.edu.cn (M.J.); peterkalds@nwafu.edu.cn (P.K.) \\ 3 Department of Animal and Poultry Production, Faculty of Environmental Agricultural Sciences, Arish \\ University, El-Arish 45511, Egypt \\ * Correspondence: xiaolongwang@nwafu.edu.cn (X.W.); chenyulin@nwafu.edu.cn (Y.C.)
}

check for updates

Citation: Ding, Q.; Jin, M.; Kalds, P.; Meng, C.; Wang, H.; Zhong, J.; Wang, X.; Chen, Y. Comparison of MicroRNA Profiles in Extracellular Vesicles from Small and Large Goat Follicular Fluid. Animals 2021, 11, 3190. https://doi.org/10.3390/ ani11113190

Academic Editor: João Pedro de Sousa Santa Clara Barbas

Received: 15 September 2021 Accepted: 5 November 2021 Published: 8 November 2021

Publisher's Note: MDPI stays neutral with regard to jurisdictional claims in published maps and institutional affiliations.

Copyright: (c) 2021 by the authors. Licensee MDPI, Basel, Switzerland. This article is an open access article distributed under the terms and conditions of the Creative Commons Attribution (CC BY) license (https:// creativecommons.org/licenses/by/ $4.0 /)$.
Simple Summary: Ovarian follicular development is associated with ovulation and is further related to litter size in goats. Extracellular vesicles (EVs) derived from miRNAs within follicular fluid undergo dynamic changes, and, together with follicle growth, may be considered as potential regulators of follicular development. However, the function and changes in EVs remain ambiguous. Here, we identified miRNA changes in EVs from small to large goat follicular fluid. Using bioinformatics tools, we demonstrated the existence of differentially expressed miRNAs in EVs from follicles of different sizes that are responsible for an altered biological effect. This study contributes to a better understanding of follicular development in goats.

Abstract: Extracellular vesicles (EVs), which exist in the follicular fluid of ruminant ovaries, are considered as cargo carriers for the transfer of biomolecules to recipient cells. However, the functions and changes in EVs in antral follicles remain ambiguous. In the present study, we isolated and characterized EVs from goat follicular fluid by means of differential ultracentrifugation and Western blotting of marker proteins. Bioinformatics tools were used to detect miRNA expression levels in EVs. Different miRNA expression patterns of EVs exist in small to large follicles. Thirteen differentially expressed miRNAs (seven upregulated and six downregulated) were identified and used for analysis. A total of 1948 predicted target genes of 13 miRNAs were mapped to signaling pathways, and three significantly enriched pathways (FoxO, MAPK, and PI3K-AKT signaling pathways) were involved in follicular development, as revealed by KEGG enrichment analysis. Our findings suggest that EVs in follicular fluid play biofunctional roles during follicular development in goats.

Keywords: extracellular vesicles; follicular fluid; small RNA sequence; miRNAs

\section{Introduction}

Multiple biological processes are involved in follicular development, including complex signaling pathways and bidirectional communication between somatic cells and oocytes [1]. Large numbers of follicles are produced in mammalian ovaries; however, only a few follicles are selected to ovulate. Typically, the follicles are divided into several stages, including the preantral and antral follicles. Large antral follicles tended to ovulate. Follicular fluid (FF), a product of blood plasma that crosses the 'blood-follicle barrier', fills the antral follicles and also contains other molecular components secreted from follicular cells [2]. The content of FF is often considered an indicator of oocyte quality and competence, containing proteins, hormones, nucleic acids (DNAs and RNAs), and other 
biomolecules similar to plasma [3]. Additionally, FF provides a perfect microenvironment for cell-to-cell communication between somatic cells and oocytes. Therefore, it is necessary to examine the content changes during follicular development.

Extracellular vesicles (EVs) are present in all bodily fluids, including ovarian FF [4,5], and have been recognized as indicators of cell communication [6]. Based on their diameter, EVs are divided into two types: exosomes ( $30-150 \mathrm{~nm})$ and microvesicles $(\sim 100-1000 \mathrm{~nm})$. In addition, EVs are often identified by protein biomarkers, such as CD 63 and TSG 101, and their morphology [7]. Recently, EVs have also been considered as cargo vessels to transfer biomolecules to recipient cells, such as DNA, RNAs (mRNA, small RNAs (sRNA), and long non-coding RNA (lncRNA), and proteins. Recent evidence has verified that EVs might support oocyte maturation [8], embryo implantation, follicular development, and other roles in reproductive processes [9]. A previous study in bovines reported that EVs from FF (FF-EVs) of small follicles significantly induced cumulus expansion compared to those from large follicles [10]. However, in some other animals, such as goats, the biofunctions of FF-EVs in reproduction are still unexplored.

EVs are often considered as natural carriers of non-coding RNAs, including microRNAs (miRNAs), which can transport their RNA content to neighboring cells or over a long distance to recipient cells by blood circulation [11]. The miRNAs are a class of approximately $22 \mathrm{nt}$ non-coding RNAs that bind to the mRNA sequence of target genes to affect protein coding that regulates biological functions. There are different types and expression levels of miRNAs in ruminant follicles during follicular development. The miRNA content in EVs is distinct in bovine follicles exposed to different estrous cycle stages [8]. Several studies have shown that extracellular miRNAs in FF from follicles of different sizes exhibit different expression profiles [7,12]. Moreover, the differentially expressed miRNAs are predicted to target genes that regulate the pathways associated with follicular development, meiotic resumption, and ovulation [13]. Therefore, we hypothesized that the expression of miRNA in EVs changes along with follicle growth and may play critical roles in the regulation of ovarian functions via miRNA transfer.

Cell-to-cell communication during ovarian follicular development involves complex molecular signaling pathways. EVs participate in these molecular processes through their secretion and uptake by follicular cells. To understand the role and importance of EVs during follicle growth in goat ovaries better, we endeavored to identify the EVs and clarify the miRNA changes in FF of small to large follicles. The findings of this study provide new insights into the content changes of goat FF and the molecular mechanisms of follicular development.

\section{Materials and Methods}

\subsection{FF Collection and EVs Isolation}

Goat ovaries were collected from Hengshan slaughterhouse (Shaanxi Province, China), which has a specialty for Shaanbei white cashmere goat in China, and transported to the laboratory in $0.9 \%$ physiological saline solution at approximately $25{ }^{\circ} \mathrm{C}$. Ovaries with obvious follicles at the surface were selected for the isolation of FF and GCs. Based on diameter, follicles were divided into two groups: small follicles $(<3 \mathrm{~mm})$ and large follicles $(>5 \mathrm{~mm}$ ) (Figure S1). FF was collected in a $1 \mathrm{~mL}$ syringe from small or large follicles, and then mixed with phosphate-buffered saline (PBS) in a 1:1 ratio. EVs were isolated using the differential ultracentrifugation method. Before ultracentrifugation, all diluted FF was spun at $300 \times g$ for 10 min to separate the cells (oocytes and GCs). Second, the supernatant FF was centrifuged at $2000 \times g$ for $20 \mathrm{~min}$ to remove the remaining dead cells and then centrifuged at $12,000 \times g$ for $45 \mathrm{~min}$ to precipitate the cellular debris and large particles, and immediately followed by filtration through a $0.22 \mu \mathrm{m}$ filter to further remove large vesicles with diameters larger than $220 \mathrm{~nm}$. Ultracentrifugation was used to isolate extracellular vesicles. Samplers were centrifuged at 110,000 $\times g$ for 90 min using an SW32Ti rotor in an Optima TMN-100XP ultracentrifuge. The pellets were resuspended in PBS and recentrifuged at $110,000 \times g$ for another $70 \mathrm{~min}$ with a Type100Ti fixed-angle rotor. All the 
mentioned centrifugations were performed at $4{ }^{\circ} \mathrm{C}$. The final $\mathrm{EV}$ pellets were suspended in PBS and stored at $-80^{\circ} \mathrm{C}$.

\subsection{Western Blotting}

The proteins of EVs in PBS buffer were isolated using RIPA lysis buffer (Beyotime, China), and supplemented with phenylmethylsulfonyl fluoride (PMSF) to a final concentration of $1 \mathrm{mM}$. The mixture was vibrated for several minutes and centrifuged at $12,000 \times \mathrm{g}$ for $10 \mathrm{~min}$. The protein concentration was quantitated using the BCA assay. The proteins CD63 and TSG101 were used to identify EVs, while calnexin (Canx, which only exists in cells) was used as a cell marker. The protein samples were mixed with $5 \times$ SDS loading buffer and boiled at $95^{\circ} \mathrm{C}$ for $5 \mathrm{~min}$. A total of $20 \mu \mathrm{g}$ of each protein sample was loaded onto a $12 \%$ SDS-PAGE gel for electrophoresis at $120 \mathrm{~V}$ for $90 \mathrm{~min}$. Proteins were transferred to polyvinylidene difluoride (PVDF) membranes (Life Technologies, Waltham, MA, USA) at a constant $260 \mathrm{~mA}$ for $2 \mathrm{~h}$. The membranes were then blocked with $5 \%(w / v)$ skim milk powder in Tris-buffered saline with $0.05 \%(v / v)$ Tween-20 (TBST) at room temperature for $2 \mathrm{~h}$. After blocking, the membranes were incubated at $4{ }^{\circ} \mathrm{C}$ overnight with primary antibodies against target proteins (anti-CD63,1:400, Cat No. 67605-1-Ig; anti-TSG101, 1:300, Cat No. 28283-1-AP; and anti-Canx 1:400, Cat No. 10427-2-AP). All primary antibodies were purchased from Proteintech (Wuhan, China) and diluted in TBST. After the primary antibodies were washed with TBST, the membranes were incubated with horseradish peroxidase-conjugated secondary anti-sheep/goat antibodies (diluted 1:2000) (Cat No. SA00001-4, Proteintech, Wuhan, China) at room temperature for $2 \mathrm{~h}$. Finally, the immunoreactive membranes were washed several times with TBST and incubated with an enhanced chemiluminescence substrate (Millipore, Billerica, MA, USA). Immunoblots were captured using an imaging system.

\subsection{Transmission Electron Microscopy (TEM) and Nanoparticle Tracking Analysis (NTA)}

To assess the morphology of EVs isolated from FF, EVs were resuspended in PBS buffer. The suspension was applied to a copper microgrid covered with a hydrophilic support film. EVs on a grid were stained with 3\% phosphotungstic acid solution for $30 \mathrm{~s}$ and desiccated in air before observation. The shapes of EVs were captured and imaged using a transmission electron microscope (TECNAI G2 SPIRIT BIO, USA) at a voltage of $80 \mathrm{kV}$. For microparticle analysis, $10 \mu \mathrm{L}$ of purified EVs were diluted in $1 \mathrm{~mL}$ PBS buffer and filtered through a $0.22 \mu \mathrm{m}$ sterile filter. Each group of EVs was prepared in three replicates, and each sample was analyzed three times. The concentration and size distribution of EVs were determined using a NanoSight NS300 (Malvern Instruments, Malvern, UK), following manufacturer protocols.

\subsection{The sRNA Extraction and sRNA Library Preparation}

Six pools of EVs (three small and three large follicle pools) were collected and used. RNAiso for sRNA (TaKaRa, Beijing, China) was used to extract sRNA from EVs. Ultracentrifuged EVs of FF were resuspended in RNAiso and the mixture was transferred to a new nuclease-free tube and vibrated thoroughly. The obtained RNAs were dissolved in nuclease-free water and stored at $-80^{\circ} \mathrm{C}$. The total RNA quantity of samples was evaluated with an Agilent 2100 Bioanalyzer (Agilent Technologies, Santa Clara, CA, USA) and RNA concentration was measured using Qubit ${ }^{\circledR}$ RNA Assay Kit in Qubit ${ }^{\circledR}$ 2.0 Fluorometer (Life Technologies, Waltham, MA, USA). A total of $20 \mathrm{ng}$ of small RNA per exosome sample was used for the sRNA sequencing library. Each sample library was constructed using the NEBNext ${ }^{\circledR}$ Multiplex Small RNA Library Prep Set for Illumina ${ }^{\circledR}$ (NEB, Ipswich, MA, USA) according to the manufacturer's recommendations. In brief, based on the special structure of the $-3^{\prime}$ and $-5^{\prime}$ ends of sRNAs, specific $3^{\prime}$ and $5^{\prime}$ adapters were directly ligated to the $3^{\prime}$ and $5^{\prime}$ ends of sRNAs, respectively. First-strand cDNA was synthesized using RNA reverse transcriptase. PCR amplification was performed using the specific primers. DNA fragments in length of 140-160 bp were purified on an 8\% PAGE gel to construct an 
sRNA library. Finally, the quality of the libraries was assessed using an Agilent Bioanalyzer 2100 system. Library construction and miRNA sequencing were performed by Novogene Company (Beijing, China).

\subsection{Small RNA Sequencing and Date Processing}

Six small RNA libraries were sequenced on an Illumina Hiseq 2500 platform, and $50 \mathrm{bp}$ single-end reads were generated. Reads containing ploy- $\mathrm{N}$, with $5^{\prime}$ adapter contaminants, without $3^{\prime}$ adapters or the insert tag, containing ploy A, T, G, or C, and low-quality reads were removed from raw data to obtain clean data. Subsequently, clean data were mapped to the reference goat genome sequence database [14] by Bowtie [15] using miRbase 20.0 as a reference. Known miRNAs were mapped to sRNA tags and modified using the software, mirdeep2. Custom scripts were used to obtain the miRNA counts and base bias on the first position of identified miRNAs of a certain length, and on each position of all identified miRNAs, respectively. To remove tags originating from protein-coding genes, repeat sequences, rRNAs, tRNAs, snRNAs, and snoRNAs, we mapped sRNA tags to the RepeatMasker or Rfam databases, or the data from cow and sheep species. To predict novel miRNAs, the characteristics of the hairpin structure of the miRNA precursor were used to explore the sRNA tags unannotated in the former steps and were integrated using the miREvo and mirdeep2 software sets. To ensure that each unique small RNA mapped to only one annotation, we followed the priority rule as follows: miRNA $>$ rRNA $>$ tRNA $>$ snRNA $>$ snoRNA $>$ novel miRNA. The miRNA expression levels were estimated by transcript per million (TPM), as previously reported [16]:

Normalized expression $=$ mapped read count $/$ total reads $\times 1,000,000$.

Differential miRNA expression analysis of the two exosome groups was performed using the DESeq package in R. The adjusted $p$-value of $<0.01$ and $\mid \log _{2}$ (fold change, FC) $\mid>1$ was set as the threshold for significantly differential expression by default.

\subsection{Target Gene Prediction and Pathways Enrichment Analyses}

The software mirDIP (http:/ / ophid.utoronto.ca/mirDIP/, accessed on 5 November 2021) was used to predict the target genes of selected miRNA with high stringency [17]. The mirDIP software contains widely used target prediction programs, including DIANA, miRDB, TargetScan, and PicTar. To verify gene prediction, miRTarbase, an experimentally validated microRNA-target interaction database, was used to further ensure target genes were obtained. Gene Ontology (GO) enrichment analysis was performed to explore the functions of the predicted target gene candidates. GO terms with adjusted $p$-values (Padj) $<0.05$, were considered significantly enriched target genes. The Kyoto Encyclopedia of Genes and Genomes (KEGG) pathways were used to annotate miRNA targets. The enrichment $p$-value was calculated using a hypergeometric distribution.

\subsection{First Strand Synthesis of $c D N A$ and Quantitative PCR ( $q P C R$ )}

Total RNA was extracted using the RNAprep Pure Micro Kit (Tiangen, Beijing, China) and reverse transcription was performed using a RevertAid First Strand cDNA synthesis kit (Thermofisher, Waltham, MA, USA) according to the manufacturer's instructions. For miRNA cDNA synthesis, stem-loop reverse primers for specific miRNAs were used. The q-PCR was performed using a $2 \times$ RealStar Green Fast Mixture (Genstar, Shanghai, China) and reaction signals were detected using a Bio-Rad CFX96 q-PCR detection system (BioRad, Hercules, CA, USA) with 96-well plates. The qPCR reaction conditions were $95^{\circ} \mathrm{C}$ for $10 \mathrm{~min}, 95^{\circ} \mathrm{C}$ for $10 \mathrm{~s}, 60^{\circ} \mathrm{C}$ for $15 \mathrm{~s}$, and $72{ }^{\circ} \mathrm{C}$ for $15 \mathrm{~s}$, followed by 40 cycles of melting curve analysis to confirm the specificity of PCR amplification.

To validate the miRNA expression data obtained from small RNA sequencing, seven miRNAs (chi-miR-202-5p, chi-miR-424-5p, chi-miR-25-3p, chi-miR-22-3p, chi-miR-26a-5p, chi-miR-191-5p, and chi-miR-126-3p) were selected for expression analysis. The miRNA Ct value was normalized to the expression level of the spiked-in cel-miR-39 using the $2^{-\Delta \Delta \mathrm{Ct}}$ 
method [18]. The stem-loop sequences and qPCR primer sequences of the miRNAs are listed in Table S2.

\subsection{Statistical Analysis}

All data are expressed as mean \pm standard error of mean (SEM) from three independent replicates for each experiment. Data from the experiments were analyzed by $t$-test or one-way analysis of variance (ANOVA) followed by Duncan's multiple range test comparisons using SPSS software (version 17.0; $p<0.05$ or $p<0.01$ indicate statistical significance.

\section{Results}

\subsection{Characterization of $E V S$ Isolated from $F F$}

The Western blot analysis revealed that EVs isolated from small and large goat FF were positive for two proteins (CD63 and TSG 101), which are well-known exosomal protein markers. Furthermore, the absence of calnexin showed the purity of EVs that were isolated using ultracentrifugation (Figure 1A). The pellet of EVs containing a bilayer membrane was observed by TEM (Figure 1B). Using nanoparticle tracking analysis (NTA), the size distribution of EVs showed that the diameter of EVs ranged from 30 to $200 \mathrm{~nm}$. There were no distinguishable changes in the size distribution of EVs between the small and large follicles (Figure 1C).
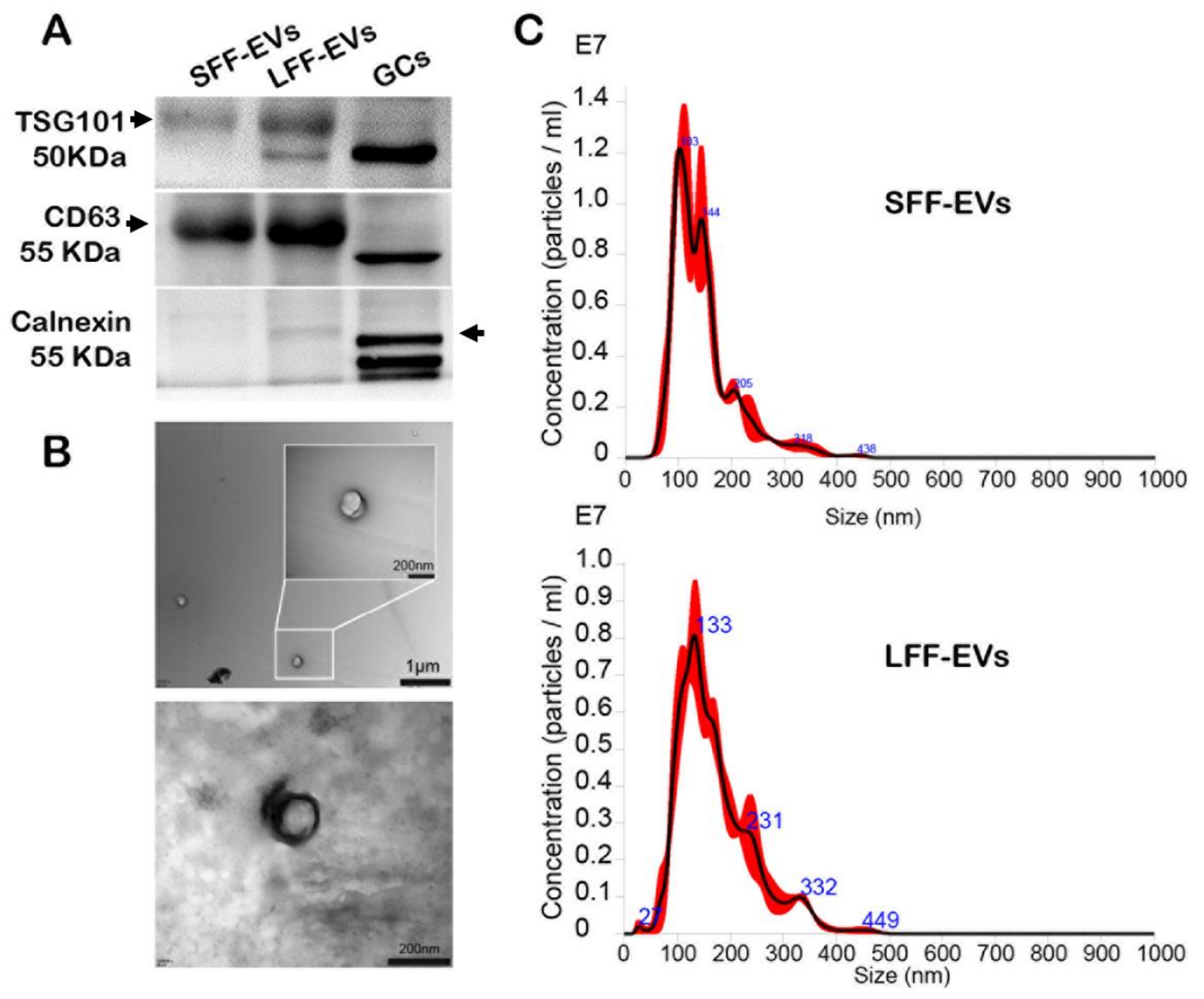

Figure 1. Identification and characterization of exosomes isolated from follicles. (A) Follicular fluidderived extracellular vesicles (EVs) were identified by means of Western blotting; (B) the morphology of EVs were observed using transmission electron microscopy (TEM; Bar = $200 \mathrm{~nm}$ ); (C) the size distribution of EVs was detected by Nanosight. SFF-EVs: small follicular fluid EVs; LFF-EVs: large follicular fluid EVs; GCs: granulosa cells. 


\subsection{The sRNA Profiles in EVs from FF of Small and Large Follicles}

3.2.1. The Sequence Length and Species Distributions of sRNA

The length of sRNAs in EVs was less than 200 nucleotides (nt) (Figure S1). This indicated that large or long nucleotide sequences might be packaged with difficulty in EVs. Six sRNA libraries of EVs were subsequently sequenced using Illumina HiSeq2000, and a mean total of 14,432,117 and 13,985,482 raw reads of LFF-EVs and SFF-EVs were obtained, respectively (Table S1). After removing redundant structures and discarding sequences shorter than $17 \mathrm{nt}$ or longer than $35 \mathrm{nt}$, a total of $13,735,702$ and 12,824,584 clean reads in LFFEVs and SFF-EVs, respectively, were determined for further analysis (Table S1). On average, in LFF-EV libraries, the length of sRNA was mostly distributed in the 20-22 nt range, while the common size of miRNAs was consistent at $22 \mathrm{nt}$ in length (Figure 2A). However, most reads in SFF-EV libraries had a high percentage of approximately $31 \mathrm{nt}$, implying more than $70 \%$ non-miRNA components (Figure 2A). The sRNA annotation showed entirely different proportions of known miRNAs, rRNA, tRNAs, snRNAs, snoRNAs, novel miRNAs, exonic RNAs, and intronic RNAs between SFF-EVs and LFF-EVs (Figure 2B). A large proportion of repeats (28.87\%) and unannotated sequences (other, 52.62\%) significantly lowered the proportion of miRNA reads in SFF-EVs (miRNA, 4.67\%) compared to LFF-EVs (miRNA $36.24 \%$, repeat $3 \%$, and other $34.85 \%$ ). This indicated that various sRNAs were loaded into EVs at different follicular stages.
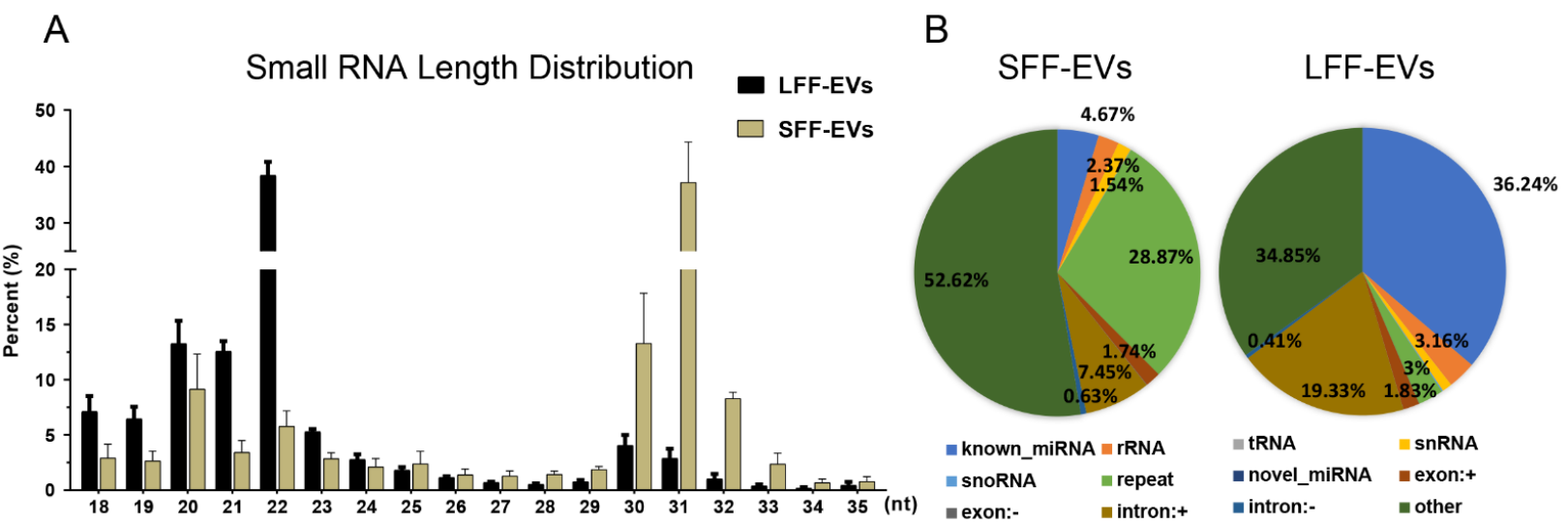

Figure 2. Overview of small RNA sequencing data. (A) Length distribution comparisons of clean reads between libraries, bars indicate the mean \pm SEM of three independent libraries, nt: nucleotides. (B) Relative abundance of different sRNA species sequenced in SFF-EVs and LFF-EVs. SFF-EVs: small follicular fluid EVs; LFF-EVs: large follicular fluid EVs.

\subsubsection{The Expression Analysis of miRNAs in EVs}

All mapped clean reads were used for further classification and assessment. A total of 288 known and 54 predicted novel miRNAs and 189 known and 28 predicted novel miRNAs were identified in LFF-EVs and SFF-EVs, respectively. Similar expression patterns were observed between the LFF-EV and SFF-EV samples (Figure 3A). Among all miRNAs, a total of 94 miRNAs, 51 upregulated miRNAs, and 43 downregulated miRNAs were significantly differentially expressed in LFF-EVs compared to SFF-EVs (Figure 3B). The heatmap shows the results of unsupervised hierarchical clustering based on the significantly differentially expressed miRNAs (Figure 3C). 

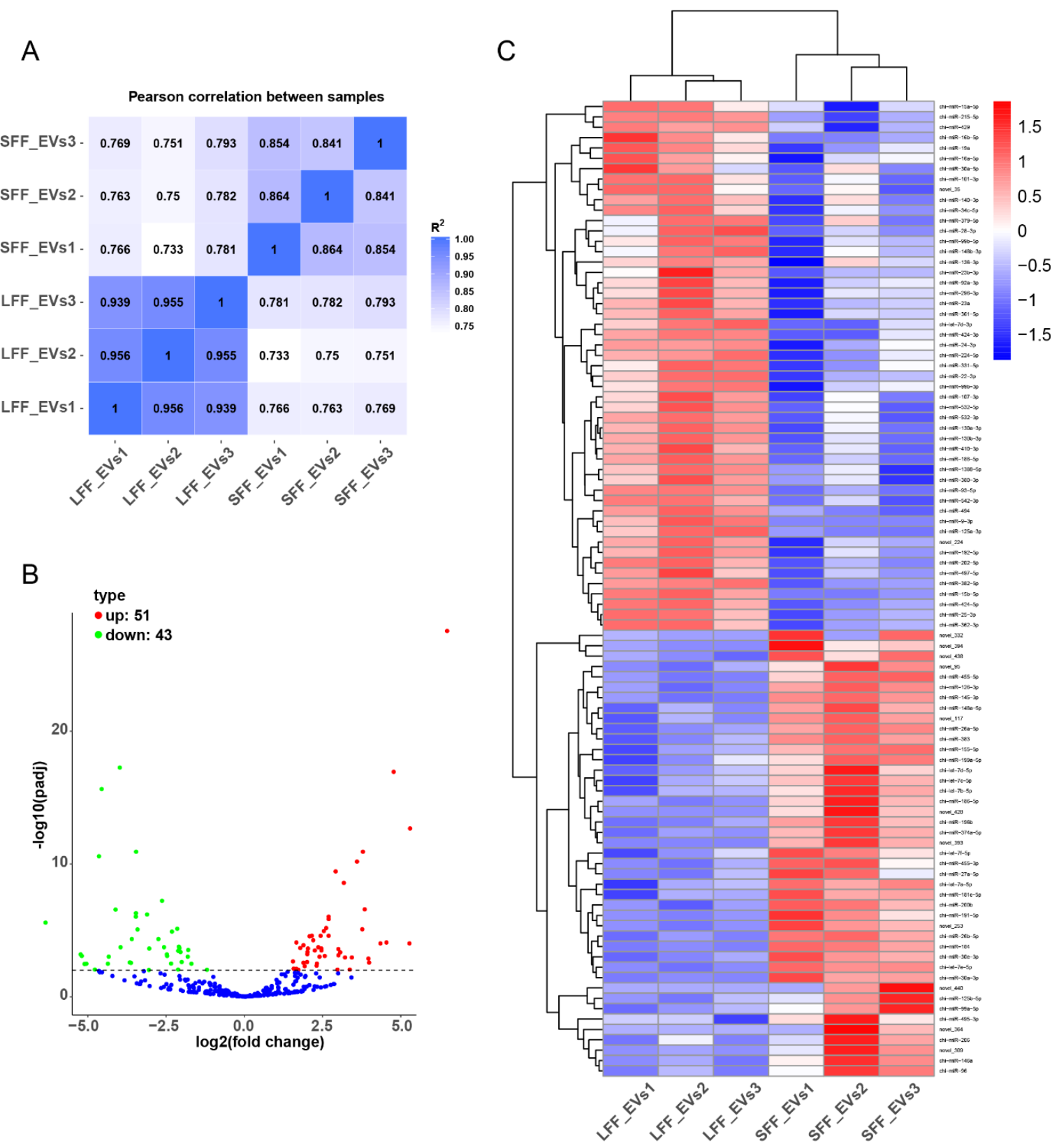

Figure 3. miRNAs expression analysis in EVs of follicular fluid (FF-EVs). (A) The Pearson correlation analysis between six libraries; (B) the volcano plot of differentially expressed miRNAs, with down-regulated miRNAs shown as green dots, whereas up-regulated miRNAs are shown as red dots; $(\mathbf{C})$ the heatmap of all differentially expressed miRNAs.

\subsection{The miRNA Profiles and Pathway Analysis of FF-EVs}

In the present study, miRNAs in EVs were ranked according to total reads normalized to transcripts per million reads (TPM). We selected miRNAs with expression levels higher than 2000 read counts; in each group, the top 20 miRNAs accounted for more than $90 \%$ of all the EV-miRNAs (Figure 4A,B). The remaining hundreds of miRNAs were present at very low read counts and occupied only a very small percentage of total reads, indicating that these miRNAs with low reads might be less likely to have significant biological functions compared to other highly expressed miRNAs in EVs. Interestingly, chi-miR-148a-5p was the most abundant miRNA, which accounted for $61.91 \%$ and $48.51 \%$ of the miRNAs in LFF-EVs and SFF-EVs, respectively (Figure 4A,B). To investigate the biological functions of miRNAs that might be involved in different stages of follicular development by follicular fluid exosome miRNA, we analyzed the biological processes and molecular pathways 
of the most expressed miRNAs from LFF-EVs or SFF-EVs. In addition, we analyzed the putative target genes of the top 20 expressed miRNAs in the two groups and identified statistically over-represented pathways of these target genes. The significant enrichment pathways were selected using a corrected $p$-value (padj) $<0.05$. The top 20 miRNAs were predicted to target 2814 and 2539 SFF-EV and LFF-EV genes, respectively (Figure 4C,D). Based on these predicted genes, similar KEGG pathway results were enriched in SFF-EVs and LFF-EVs. However, the insulin signaling pathway and progesterone-mediated oocyte maturation were specifically predicted in SFF-EVs. This implies that the EV-miRNAs play different roles during follicular development.

A
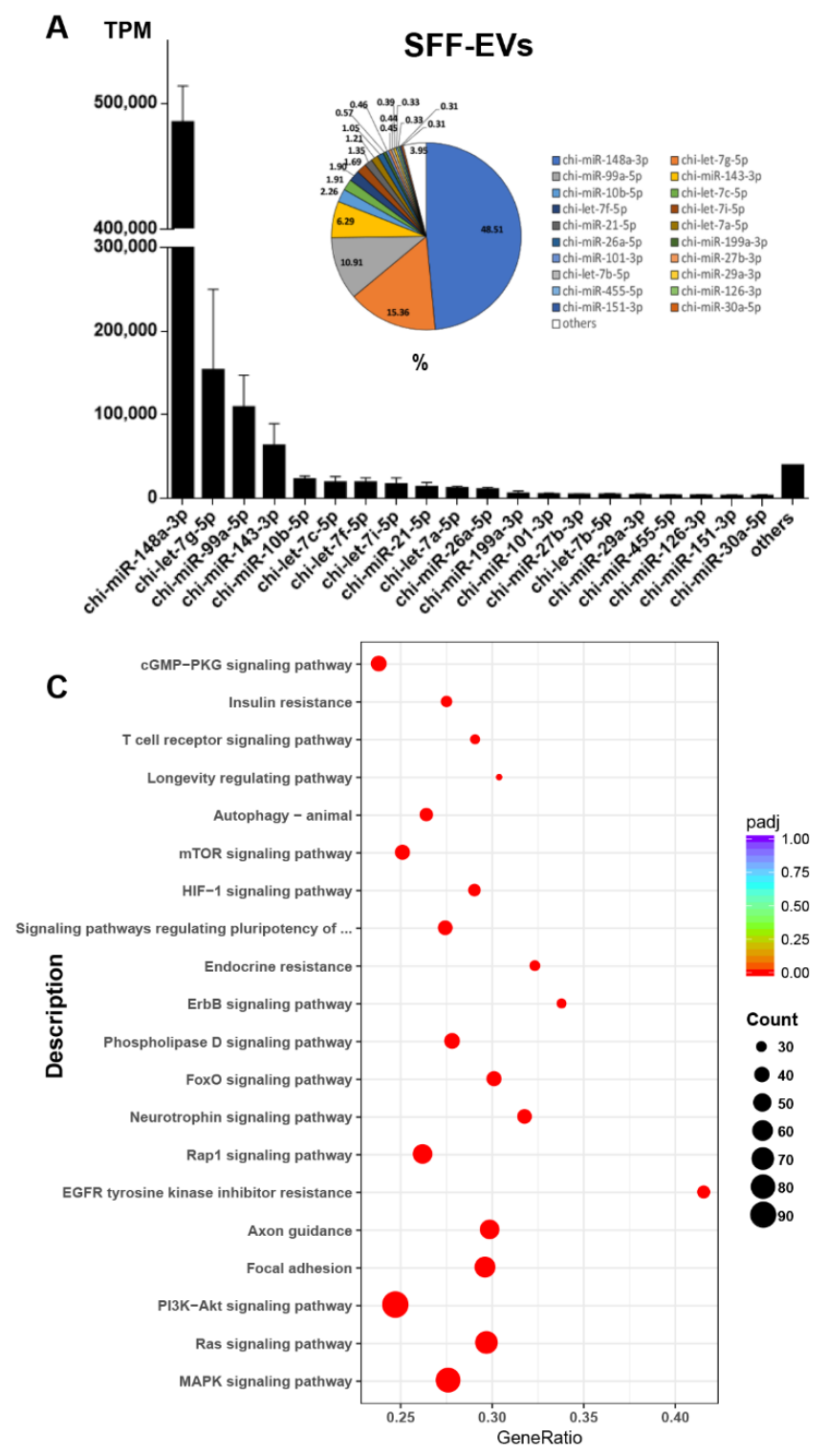

B

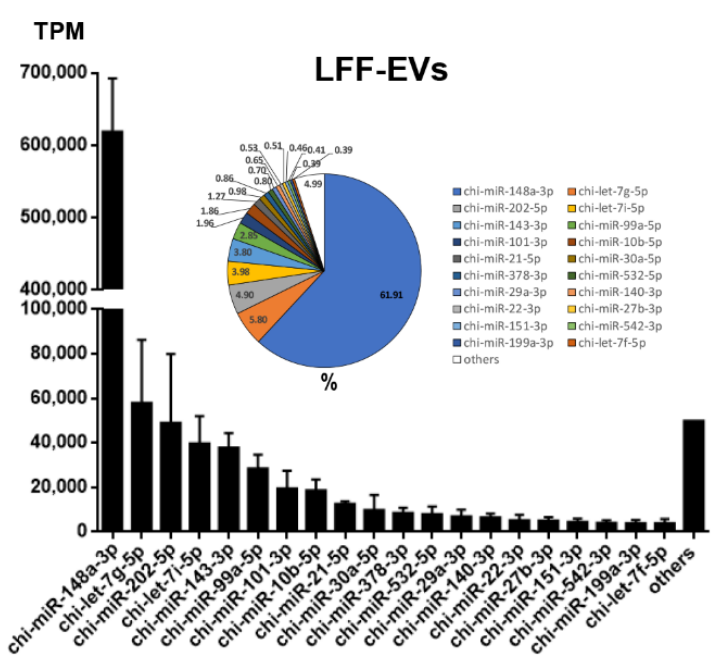

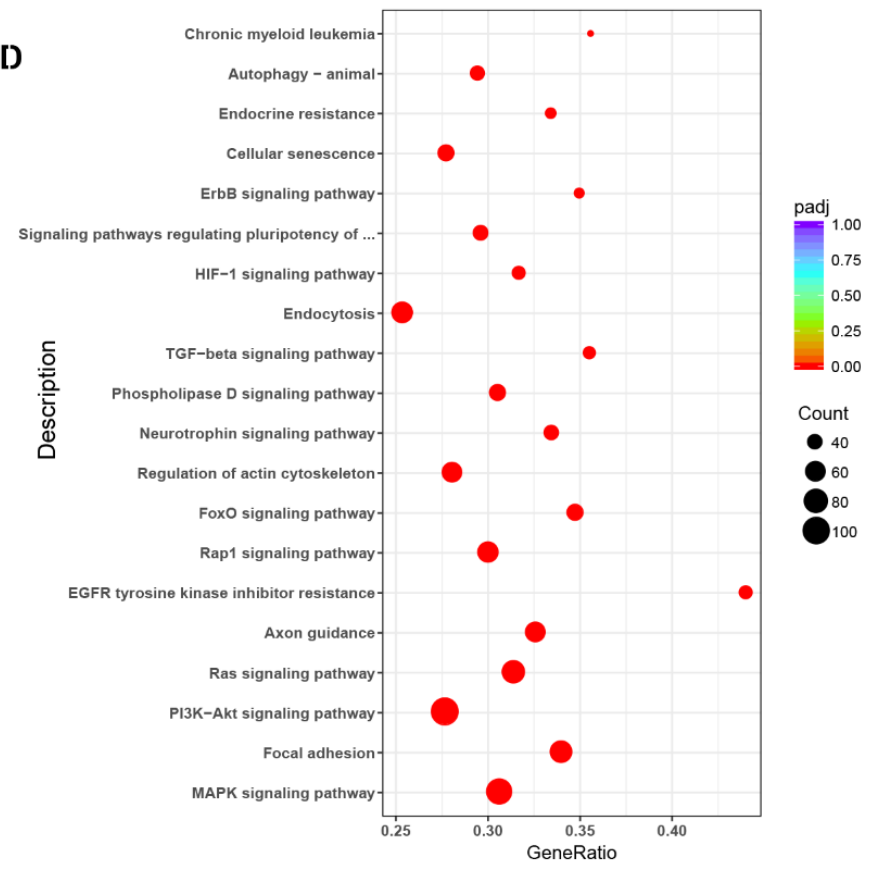

Figure 4. Expression distribution and predicted pathways of target genes of most enriched miRNAs. (A,B) The distribution of top 20 most expressed miRNAs in SFF-EVs and LFF-EVs; (C,D) the most relevant pathways regulated by the top 20 most expressed miRNAs identified in SFF-EVs and LFF-EVs, padj: corrected $p$-value.

In order to determine gene functions in cellular and biological pathways that are potentially regulated by exosomal miRNAs in follicular development, we performed pathway analysis of predicted genes of miRNAs. Regardless of the low expression of differentially expressed miRNAs, we focused on the high expression of miRNAs in the two experimental groups. A total of seven upregulated (chi-miR-202-5p, chi-miR-22-3p, 
chi-miR-542-3p, chi-miR-532-5p, chi-miR-101-3p, chi-miR-30a-5p, and chi-miR-140-3p) and six downregulated (chi-let-7f-5p, chi-miR-99a-5p, chi-miR-455-5p, chi-miR-126-3p, chi-let-7a-5p, and chi-miR-26a-5p) miRNAs in LFF-EVs were determined (Table 1).

Table 1. The most significantly differently expressing miRNAs.

\begin{tabular}{ccccc}
\hline sRNA & $\begin{array}{c}\log _{2} \text { Fold Change } \\
\text { (LFF-EVs/SFF-EVs) }\end{array}$ & $p$-Value & $\begin{array}{c}\text { Adjusted } \\
p \text {-Value }\end{array}$ & Regulation \\
\hline chi-miR-202-5p & 6.4674 & $8.44 \times 10^{-31}$ & $2.85 \times 10^{-28}$ & $\mathrm{Up}$ \\
chi-miR-22-3p & 3.5869 & $1.98 \times 10^{-12}$ & $6.68 \times 10^{-11}$ & $\mathrm{Up}$ \\
chi-miR-542-3p & 3.1663 & $9.48 \times 10^{-11}$ & $2.67 \times 10^{-9}$ & $\mathrm{Up}$ \\
chi-miR-532-5p & 2.1443 & $2.28 \times 10^{-6}$ & $2.57 \times 10^{-5}$ & $\mathrm{Up}$ \\
chi-miR-101-3p & 2.0369 & $5.97 \times 10^{-6}$ & $1.41 \times 10^{-4}$ & $\mathrm{Up}$ \\
chi-miR-30a-5p & 2.0034 & $6.47 \times 10^{-4}$ & $5.55 \times 10^{-3}$ & $\mathrm{Up}$ \\
chi-miR-140-3p & 1.3427 & $1.18 \times 10^{-5}$ & $2.58 \times 10^{-4}$ & $\mathrm{Up}$ \\
chi-let-7f-5p & -1.7542 & $2.75 \times 10^{-3}$ & $1.57 \times 10^{-2}$ & Down \\
chi-miR-99a-5p & -1.846 & $3.73 \times 10^{-4}$ & $3.77 \times 10^{-3}$ & Down \\
chi-miR-455-5p & -2.1587 & $5.42 \times 10^{-7}$ & $7.64 \times 10^{-6}$ & Down \\
chi-miR-126-3p & -3.4743 & $2.87 \times 10^{-13}$ & $1.21 \times 10^{-11}$ & Down \\
chi-let-7a-5p & -3.4784 & $2.43 \times 10^{-8}$ & $5.12 \times 10^{-7}$ & Down \\
chi-miR-26a-5p & -3.9945 & $3.28 \times 10^{-20}$ & $5.54 \times 10^{-18}$ & Down \\
\hline
\end{tabular}

For these 13 miRNAs, a total of 1948 target genes were predicted, and the most enriched terms were provided based on Gene Ontology (GO) analysis results. The 10 most enriched terms in every catalog (biological process, BB; cellular component, CC; and molecular function, MF) are shown in Figure 5A. Biological regulation, membranes, and protein binding were significantly enriched in the three catalogs (BB, CC, and MF). Additionally, the number of KEGG pathways was significantly enriched by the predicted genes. Here, we identified the top 20 pathways that were most significantly enriched. The targets were mainly involved in axon guidance, the FoxO signaling pathway, the MAPK signaling pathway, and the PI3K-AKT signaling pathway, inter alia (Figure 5B). The network of miRNAs and their target genes involved in the above pathways showed a complex relationship between miRNA and target genes (Figure 5C). Collectively, our results provide insight into the involvement of miRNAs in FF-EVs through several signaling pathways. 


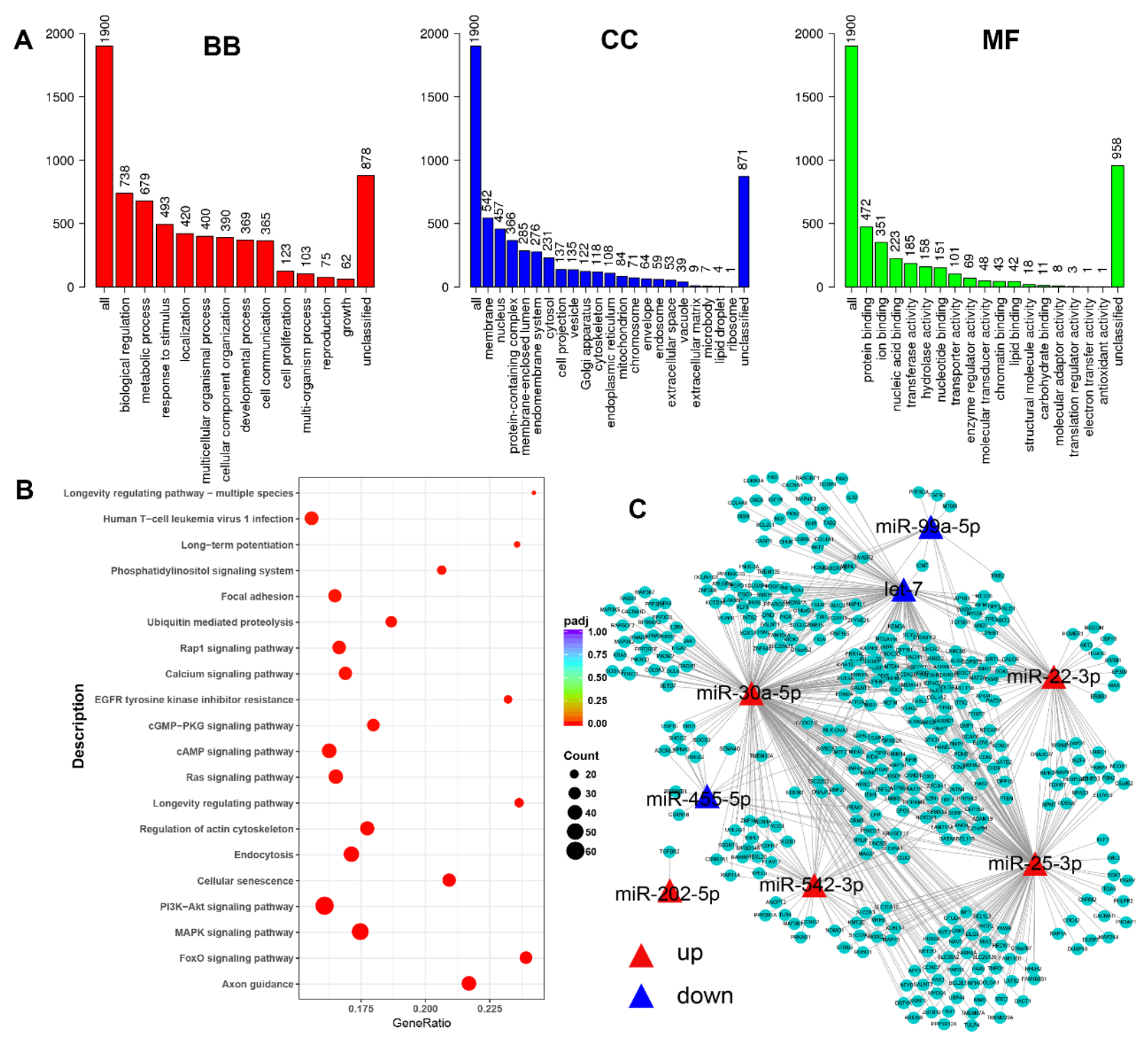

Figure 5. Bioinformatics analysis of differentially expressed miRNAs. (A) The Gene Ontology (GO) analysis of differentially expressed miRNAs predict target genes, three catalogues in different colors (biological process (BB), cellular component (CC) and molecular function (MF)); (B) the most enriched Kyoto Encyclopedia of Genes and Genomes (KEGG) pathways of predicted target genes, padj: adjust $p$-value; (C) the network of miRNA-genes of most related pathways.

\subsection{Validation of Sequencing Results Using RT-qPCR}

The expression of seven upregulated and downregulated miRNAs was randomly selected to verify miRNA sequencing data. An accurate content of synthetic miRNA, cel-miR-39, as a normalized miRNA, was added to the total miRNA of exosomal LFF-EVs or SFF-EVs before first-strand cDNA synthesis. The results of the qPCR analysis show that the expression of these seven miRNAs was consistent with that obtained using RNA-seq (Figure 6). These data confirm that the sequencing data were reliable and accurate. 


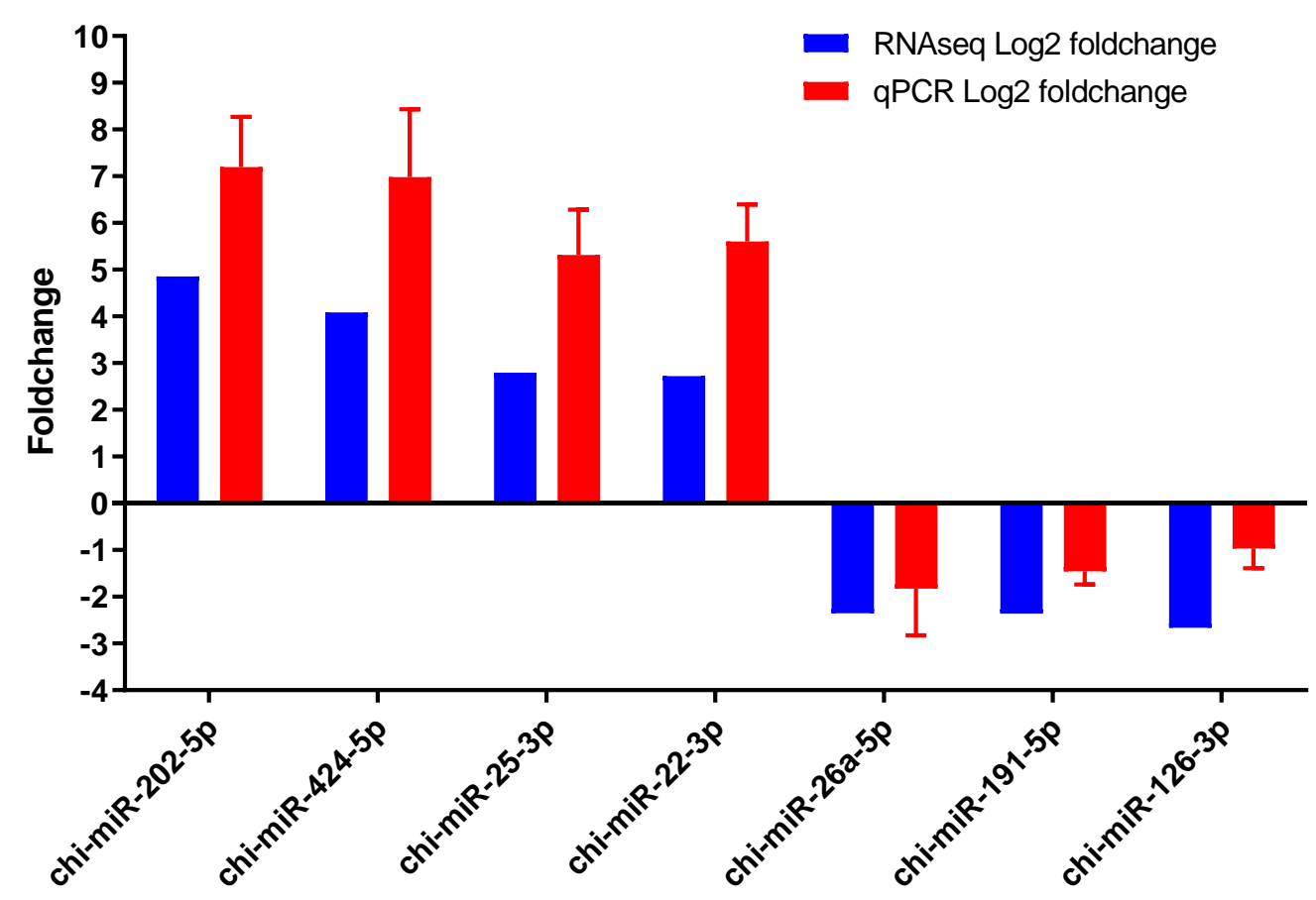

Figure 6. Validation of small RNA sequencing results by qPCR. Experiments were repeated three times. Bars indicate the mean \pm SEM of three independent replicates.

\section{Discussion}

Goat follicular growth is stimulated by gonadal hormones during the estrous cycle. Ovarian follicles provide a complex microenvironment for the interaction between somatic cells and oocytes. The follicular fluid composition shows dynamic changes during follicular growth and maturation. In the current study, we reported extremely different sRNA profiles in EVs between small and large follicles in goats, including sRNA sequence length distributions, relative abundance of different RNA species, and miRNA expression levels. To our knowledge, sRNA loading into EVs depends on the affinity of sRNA interacting with the EV membrane, which involves the sRNA sorting process [19]. This indicates a potentially unexplored change in EVs during follicular development.

The miRNA expression patterns were significantly different between SFF-EVs and LFF-EVs. Here, we accounted for the miRNA concentrations of EVs and showed that the 20 most enriched miRNAs in each group accounted for more than $90 \%$ of all miRNAs, since the remaining miRNAs formed only a small percentage of all miRNAs. The miRNAs are packaged into EVs, and it has been reported that low-abundance miRNAs (even less than one copy) load into a single EV [20]. It is difficult to accurately evaluate the function of the miRNAs with lower expression levels. Therefore, in this study, we focused on the different expression levels of the most enriched miRNAs in the two groups.

The miRNAs profiles in follicular fluid EVs have been reported in different species by comparing the most enriched miRNAs in bovine [21], humans [22], horses [23], pigs [24] and goats. Some abundant miRNAs, such as miR-202-5p, miR-99a, miR-21-5p and miR$29 a-5 p$, are shared in pigs and LFF-EVs of goats. The other species are different from each other. Different miRNAs profiles in EVs may show different mechanisms of actions in follicular cells in different species. In bovine, after incubation with follicular fluid, exosomes promoted cumulus expansion and oocytes maturation in vitro [10], while this was not observed in pigs [25].

The FoxO signaling pathway, MAPK signaling pathway, and PI3K-Akt signaling pathway were the most enriched by the target genes of 13 differentially expressed miRNAs. These signaling pathways play key roles in the proliferation and apoptosis of mammalian cells during follicular growth and in the promotion of primordial follicle activation [26,27]. 
The PI3K-Akt pathway is regulated by several miRNAs, such as miR-202-5p [28], miR$101[29,30]$, and miR-22-3p [31], which are upregulated in LFF-EVs. It was reported that exosomes promote GCs' proliferation through the P13K-AKT signaling [12] and MAPK signaling pathways [24]. In addition, miR-542-5p was previously found to be upregulated in GCs from bovine pre-ovulatory follicles compared to those in subordinate follicles [32]. Specifically, miR-202-5p is most significantly upregulated in LFF-EVs compared to SFF-EVs. In ruminants, miR-202-5p is highly expressed in large follicles in goats [33] and cattle [34]. In lower-order vertebrates, miR-202-5p knock-out causes the early steps of medaka oogenesis/folliculogenesis impairment and reduces the number of large follicles, leading to low fertility [35]. Moreover, miR-202-5p has been identified as a germline-specific miRNA [36-38] and can regulate ovarian hormone metabolism [39] and GC proliferation [40]. Thus, the biofunction of miR-202-5p in follicular development should be further explored. In mammalian ovaries, a large number of genes and related pathways participate in ovarian follicular growth until ovulation. Therefore, the molecular functions of predicted targets and pathways of miRNAs require further experimental verification.

In summary, we isolated and characterized EVs from the small and large follicles of goats. We revealed distinct EV-miRNA profiles from small to large follicles that are associated with follicular growth. Seven miRNAs with high levels were upregulated in large follicle EVs compared to small follicle EVs. Of these, miR-202-5p was shown as a follicle development potential marker packaged into EVs. Our study revealed that the EVs and their miRNA cargos changed dynamically over time, suggesting their potential use as biomarkers for cell-to-cell communication during follicular development. Finally, this study shows a specific FF-EV component and a set of miRNAs that may play key roles during follicle growth in goat ovaries.

Supplementary Materials: The following are available online at https:/ /www.mdpi.com/article/ 10.3390/ani11113190/s1, Figure S1. Small or large follicles selected from goat ovaries; Figure S2. Length distribution of small RNAs in EVs; Table S1. Summary of sRNA data output and filter; Table S2. miRNA primer sequences used in this study.

Author Contributions: Q.D., X.W. and Y.C. designed the research; Q.D. and M.J. performed the experiments, Q.D., M.J., P.K. and X.W. analyzed the data and wrote the manuscript. Original draft revision, Q.D., P.K., C.M., H.W. and J.Z. Funding acquisition, Y.C. and Q.D. All authors have read and agreed to the published version of the manuscript.

Funding: This work was funded by the Natural Science Foundation of China (32102550), the National Key Research and Development Program of China (2018YFD0501905), and China Agriculture Research System (CARS-39-12).

Institutional Review Board Statement: All studies were conducted in accordance with the guidelines approved by the Experimental Animal Committee of the Northwest A\&F University, Yangling, China (Approval ID: 2014ZX08008-002).

Informed Consent Statement: Not applicable.

Data Availability Statement: Qiang Ding had full access to all the data in this study and takes responsibility for the integrity of the data and the accuracy of the data analysis. The data that support the findings of this study are available from the corresponding author upon reasonable request.

Acknowledgments: We thank Fangxiong Shi (Nanjing Agricultural University) for writing advice.

Conflicts of Interest: The authors declare no conflict of interest. 


$\begin{array}{ll}\text { Abbreviations } & \\ \text { BB } & \text { Biological Process } \\ \text { CC } & \text { Cellular Component } \\ \text { circRNA } & \text { circular RNA } \\ \text { COC } & \text { Cumulus-Oocyte Complex } \\ \text { EVs } & \text { Extracellular vesicles } \\ \text { FF } & \text { Follicular fluids } \\ \text { FoxO } & \text { Forkhead box O } \\ \text { GCs } & \text { Granulosa cells } \\ \text { GO } & \text { Gene Ontology } \\ \text { KEGG } & \text { Kyoto Encyclopedia of Genes and Genomes } \\ \text { lncRNA } & \text { long non-coding RNA } \\ \text { MAPK pathway } & \text { mitogen-activated protein kinase pathway } \\ \text { MF } & \text { Molecular Function } \\ \text { miRNA } & \text { microRNA } \\ \text { NTA } & \text { Nanoparticle Tracking Analysis } \\ \text { PI3K-AKT } & \text { phosphoinositide 3-kinase Active } \\ \text { piRNA } & \text { Piwi-interacting RNA } \\ \text { PMSF } & \text { Phenylmethylsulfonyl Fluoride } \\ \text { rRNA } & \text { Ribosomal RNA } \\ \text { snoRNA } & \text { small nucleolar RNA } \\ \text { snRNA } & \text { small nuclearRNA } \\ \text { TEM } & \text { Transmission electron microscopy } \\ \text { tRNA } & \text { Transfer RNA } \\ \text { BB } & \text { Biological Process } \\ & \end{array}$

\section{References}

1. Eppig, J.J. Oocyte control of ovarian follicular development and function in mammals. Reproduction 2001, 122, 829. [CrossRef]

2. Gosden, R.G.; Hunter, R.H.; Telfer, E.; Torrance, C.; Brown, N. Physiological factors underlying the formation of ovarian follicular fluid. J. Reprod. Fertil. 1988, 82, 813-825. [CrossRef]

3. Alberto, R.; Luisa, D.P.; Simona, C.; Emanuela, M.; Marco, M.; Paolo, R. Follicular fluid content and oocyte quality: From single biochemical markers to metabolomics. Reprod. Biol. Endocrinol. 2009, 7, 40.

4. Pietro, C.D. Exosome-mediated communication in the ovarian follicle. J. Assist. Reprod. Genet. 2016, 33, 303-311. [CrossRef]

5. Saeedzidane, M.; Linden, L.; Salilewwondim, D.; Held, E.; Neuhoff, C.; Tholen, E.; Hoelker, M.; Schellander, K.; Tesfaye, D. Cellular and exosome mediated molecular defense mechanism in bovine granulosa cells exposed to oxidative stress. PLoS ONE 2017, 12, e0187569.

6. Tkach, M.; Théry, C. Communication by Extracellular Vesicles: Where We Are and Where We Need to Go. Cell 2016, 164, 1226-1232. [CrossRef]

7. Navakanitworakul, R.; Hung, W.T.; Gunewardena, S.; Davis, J.S.; Chotigeat, W.; Christenson, L.K. Characterization and Small RNA Content of Extracellular Vesicles in Follicular Fluid of Developing Bovine Antral Follicles. Sci. Rep. 2016, 6, 25486. [CrossRef]

8. de Ávila, A.C.F.C.M.; Bridi, A.; Andrade, G.M.; del Collado, M.; Sangalli, J.R.; Nociti, R.P.; da Silva Junior, W.A.; Bastien, A.; Robert, C.; Meirelles, F.V.; et al. Estrous cycle impacts miRNA content in extracellular vesicles that modulate bovine cumulus cell transcripts during in vitro maturation. Biol. Reprod. 2019, 102, 362-375. [CrossRef] [PubMed]

9. Machtinger, R.; Laurent, L.C.; Baccarelli, A.A. Extracellular vesicles: Roles in gamete maturation, fertilization and embryo implantation. Hum. Reprod. Update 2016, 22, 182. [CrossRef] [PubMed]

10. Hung, W.T.; Hong, X.; Christenson, L.K.; Mcginnis, L.K. Extracellular Vesicles from Bovine Follicular Fluid Support Cumulus Expansion. Biol. Reprod. 2015, 93, 117. [CrossRef] [PubMed]

11. Fatima, F.; Nawaz, M. Long Distance Metabolic Regulation through Adipose-Derived Circulating Exosomal miRNAs: A Trail for RNA-Based Therapies? Front. Physiol. 2017, 8, 545. [CrossRef]

12. Hung, W.T.; Navakanitworakul, R.; Khan, T.; Davis, P.; Mcginnis, L.K.; Christenson, L.K. Stage-specific follicular extracellular vesicle uptake and regulation of bovine granulosa cell proliferation. Biol. Reprod. 2017, 49, 123. [CrossRef] [PubMed]

13. Machtinger, R.; Rodosthenous, R.S.; Adir, M.; Mansour, A.; Racowsky, C.; Baccarelli, A.A.; Hauser, R. Extracellular microRNAs in follicular fluid and their potential association with oocyte fertilization and embryo quality: An exploratory study. J. Assist. Reprod. Genet. 2017, 34, 1-9. [CrossRef]

14. Dong, Y.; Xie, M.; Jiang, Y.; Xiao, N.; Du, X.; Zhang, W.; Tosser-Klopp, G.; Wang, J.; Yang, S.; Liang, J.; et al. Sequencing and automated whole-genome optical mapping of the genome of a domestic goat (Capra hircus). Nat. Biotechnol. 2013, 31, 135-141. [CrossRef] [PubMed]

15. Langmead, B.; Trapnell, C.; Pop, M.; Salzberg, S.L. Ultrafast and memory-efficient alignment of short DNA sequences to the human genome. Genome Biol. 2009, 10, R25. [CrossRef] 
16. Zhou, L.; Chen, J.; Li, Z.; Li, X.; Hu, X.; Yi, H.; Zhao, X.; Liang, C.; Wang, Y.; Sun, L. Integrated Profiling of MicroRNAs and mRNAs: MicroRNAs Located on Xq27.3 Associate with Clear Cell Renal Cell Carcinoma. PLoS ONE 2010, 5, e15224. [CrossRef]

17. Tokar, T.; Pastrello, C.; Rossos, A.E.M.; Abovsky, M.; Hauschild, A.C.; Tsay, M.; Lu, R.; Jurisica, I. mirDIP 4.1-integrative database of human microRNA target predictions. Nucleic Acids Res. 2018, 46, D360-D370. [CrossRef] [PubMed]

18. Livak, K.J.; Schmittgen, T.D. Analysis of Relative Gene Expression Data Using Real-Time Quantitative PCR and the 2- $\Delta \Delta C T$ Method. Methods 2001, 25, 402-408. [CrossRef] [PubMed]

19. Janas, T.; Janas, M.M.; Sapon, K.; Janas, T. Mechanisms of RNA loading into exosomes. FEBS Lett. 2015, 589, 1391-1398. [CrossRef]

20. Chevillet, J.R.; Kang, Q.; Ruf, I.K.; Briggs, H.A.; Vojtech, L.N.; Hughes, S.M.; Cheng, H.H.; Arroyo, J.D.; Meredith, E.K.; Gallichotte, E.N.; et al. Quantitative and stoichiometric analysis of the microRNA content of exosomes. Proc. Natl. Acad. Sci. USA 2014, 111, 14888-14893. [CrossRef]

21. Sohel, M.M.H.; Hoelker, M.; Noferesti, S.S.; Salilewwondim, D.; Tholen, E.; Looft, C.; Rings, F.; Uddin, M.J.; Spencer, T.E.; Schellander, K. Exosomal and Non-Exosomal Transport of Extra-Cellular microRNAs in Follicular Fluid: Implications for Bovine Oocyte Developmental Competence. PLoS ONE 2013, 8, e78505.

22. Santonocito, M.; Vento, M.; Guglielmino, M.R.; Battaglia, R.; Wahlgren, J.; Ragusa, M.; Barbagallo, D.; Borzì, P.; Rizzari, S.; Maugeri, M. Molecular characterization ofexosomes and their microRNA cargo in human follicular fluid: Bioinformatic analysis reveals that exosomal microRNAs control pathways involved in follicular maturation. Fertil. Steril. 2014, 102, $1751-1761$. [CrossRef]

23. Silveira, J.; Winger, Q.A.; Bouma, G.J.; Carnevale, E.M. Effects of age on follicular fluid exosomal microRNAs and granulosa cell transforming growth factor- $\beta$ signalling during follicle development in the mare. Reprod. Fertil. Dev. 2015, 27, 897. [CrossRef] [PubMed]

24. Yuan, C.; Li, Z.; Zhao, Y.; Wang, X.; Chen, L.; Zhao, Z.; Cao, M.; Chen, T.; Iqbal, T.; Zhang, B.; et al. Follicular fluid exosomes: Important modulator in proliferation and steroid synthesis of porcine granulosa cells. FASEB J. 2021, 35, e21610. [CrossRef] [PubMed]

25. Matsuno, Y.; Onuma, A.; Fujioka, Y.A.; Yasuhara, K.; Fujii, W.; Naito, K.; Sugiura, K. Effects of exosome-like vesicles on cumulus expansion in pigs in vitro. J. Reprod. Dev. 2017, 63, 51-58. [CrossRef] [PubMed]

26. Grosbois, J.; Demeestere, I. Dynamics of PI3K and Hippo signaling pathways during in vitro human follicle activation. Hum. Reprod. 2018, 33, 1705-1714. [CrossRef]

27. Lu, X.; Guo, S.; Cheng, Y.; Kim, J.H.; Feng, Y.; Feng, Y. Stimulation of ovarian follicle growth after AMPK inhibition. Reproduction 2017, 153, 683-694. [CrossRef]

28. Liu, T.; Guo, J.; Zhang, X. MiR-202-5p/PTEN mediates doxorubicin-resistance of breast cancer cells via PI3K/Akt signaling pathway. Cancer Biol. 2019, 20, 989-998. [CrossRef]

29. An, X.; Ma, H.; Liu, Y.; Li, F.; Song, Y.; Li, G.; Bai, Y.; Cao, B. Effects of miR-101-3p on goat granulosa cells in vitro and ovarian development in vivo via STC1. J. Anim. Sci. Biotechnol. 2020, 11, 102. [CrossRef]

30. Sachdeva, M.; Wu, H.; Ru, P.; Hwang, L.; Trieu, V.; Mo, Y.Y. MicroRNA-101-mediated Akt activation and estrogen-independent growth. Oncogene 2011, 30, 822-831. [CrossRef]

31. Wang, X.; Wang, Y.; Kong, M.; Yang, J. MiR-22-3p suppresses sepsis-induced acute kidney injury by targeting PTEN. Biosci. Rep. 2020, 40. [CrossRef]

32. Gebremedhn, S.; Salilew-Wondim, D.; Ahmad, I.; Sahadevan, S.; Hossain, M.M.; Hoelker, M.; Rings, F.; Neuhoff, C.; Tholen, E.; Looft, C.; et al. MicroRNA Expression Profile in Bovine Granulosa Cells of Preovulatory Dominant and Subordinate Follicles during the Late Follicular Phase of the Estrous Cycle. PLoS ONE 2015, 10, e0125912. [CrossRef]

33. Lu, T.; Zou, X.; Liu, G.; Deng, M.; Sun, B.; Guo, Y.; Liu, D.; Li, Y. A Preliminary Study on the Characteristics of microRNAs in Ovarian Stroma and Follicles of Chuanzhong Black Goat during Estrus. Genes 2020, 11, 970. [CrossRef]

34. Sontakke, S.D.; Mohammed, B.T.; Mcneilly, A.S.; Donadeu, F.X. Characterization of microRNAs differentially expressed during bovine follicle development. Reproduction 2014, 148, 271-283. [CrossRef]

35. Stéphanie, G.; Jérôme, B.; Amine, B.; Laure, H.; Clara, D.; Fabrice, L.; Jérôme, M.; Aurélie, L.C.; Anne, S.; Julien, B. MiR-202 controls female fecundity by regulating medaka oogenesis. PLOS Genet. 2018, 14, e1007593.

36. Wainwright, E.N.; Jorgensen, J.S.; Kim, Y.; Truong, V.; Bagheri-Fam, S.; Davidson, T.; Svingen, T.; Fernandez-Valverde, S.L.; McClelland, K.S.; Taft, R.J.; et al. SOX9 Regulates MicroRNA miR-202-5p/3p Expression During Mouse Testis Differentiation. Biol. Reprod. 2013, 89, 2. [CrossRef] [PubMed]

37. Chen, J.; Cai, T.; Zheng, C.; Lin, X.; Wang, G.; Liao, S.; Wang, X.; Gan, H.; Zhang, D.; Hu, X. MicroRNA-202 maintains spermatogonial stem cells by inhibiting cell cycle regulators and RNA binding proteins. Nucleic Acids Res. 2017, 45, 4142. [CrossRef] [PubMed]

38. Zhang, J.; Liu, W.; Jin, Y.; Jia, P.; Jia, K.; Yi, M. MiR-202-5p is a novel germ plasm-specific microRNA in zebrafish. Sci. Rep. 2017, 7, 7055. [CrossRef] [PubMed]

39. Sørensen, A.E.; Udesen, P.B.; Maciag, G.; Geiger, J.; Saliani, N.; Januszewski, A.S.; Jiang, G.; Ma, R.C.; Hardikar, A.A.; Wissing, M.L.M.; et al. Hyperandrogenism and Metabolic Syndrome Are Associated with Changes in Serum-Derived microRNAs in Women with Polycystic Ovary Syndrome. Front. Med. 2019, 6, 242. [CrossRef]

40. Ding, Q.; Jin, M.; Wang, Y.; Liu, J.; Chen, Y. Transactivation of miR-202-5p by Steroidogenic Factor 1 (SF1) Induces Apoptosis in Goat Granulosa Cells by Targeting TGF $\beta$ R2. Cells 2020, 9, 445. [CrossRef] 